\title{
Spreading depolarization may represent a novel mechanism for delayed fluctuating neurological deficit after chronic subdural hematoma evacuation
}

\author{
Laila M. Mohammad, MD, Mohammad Abbas, MD, ${ }^{2}$ C. William Shuttleworth, $\mathrm{PhD},{ }^{3}$ \\ Rosstin Ahmadian, BS, ${ }^{4}$ Annapoorna Bhat, MD, PhD, ${ }^{2}$ Deirdre A. Hill, $\mathrm{PhD},{ }^{5}$ and \\ Andrew P. Carlson, MD, MS-CR ${ }^{1}$
}

Departments of ${ }^{1}$ Neurosurgery, ${ }^{2}$ Neurology, ${ }^{3}$ Neurosciences, and ${ }^{5}$ Internal Medicine, University of New Mexico School of Medicine; and ${ }^{4}$ University of New Mexico School of Medicine, Albuquerque, New Mexico

\begin{abstract}
OBJECTIVE Most patients with chronic subdural hematoma (CSDH) recover after surgical evacuation with a straightforward course. There is a subset of patients who develop transient and fluctuating deficits not explained by seizures, stroke, or mass effect after evacuation. The objective of this study was to investigate whether these postoperative neurological deficits may be related to temporary brain dysfunction caused by cortical spreading depolarizations (SDs).

METHODS The authors conducted a prospective observational study of 40 patients who underwent cSDH evacuation. At the time of surgery, a $1 \times 6$ subdural electrode strip was placed on the cortex parallel to the subdural drain. Clinical outcomes were assessed utilizing the Markwalder Grading Scale, need for clinical EEG for new deficit, and presence of new deficits.
\end{abstract}

RESULTS Definitive SD was detected in 6 (15\%) of 40 patients. Baseline and CSDH characteristics did not differ between patients with and without SD. More patients experienced postoperative neurological deterioration if they had SD $(50 \%)$ compared to those without SD $(8.8 \% ; p=0.03)$. Only 2 patients in the entire cohort demonstrated early neurological deterioration, both of whom had SD. One of these cases demonstrated a time-locked new focal neurological deficit (aphasia) at the start of a series of multiple clusters of SD.

CONCLUSIONS This is the first observation of SD occurring after CSDH evacuation. SD occurred at a rate of $15 \%$ and was associated with neurological deterioration. This may represent a novel mechanism for otherwise unexplained fluctuating neurological deficit after CSDH evacuation. This could provide a new therapeutic target, and SD-targeted therapies should be evaluated in prospective clinical trials.

https://thejns.org/doi/abs/10.3171/2020.1.JNS192914

KEYWORDS chronic subdural hematoma; cortical spreading depolarizations; seizure; spreading depression; transient neurological deficit; trauma

$\sim_{i}$ HRONIC subdural hematoma (cSDH) is one of the most common pathologies encountered by practicing neurosurgeons. ${ }^{1,2}$ The incidence of $\mathrm{cSDH}$ is 1.7 per 100,000 in the general population and increases to 286 per 100,000 in patients 80 years of age or older. Hospitalizations from 1998 compared to 2007 have increased from 30.2 per 100,000 to 41.9 per $1000,{ }^{2}$ and $\mathrm{cSDH}$ has been estimated to become the most common condition treated by neurosurgeons by $2030 .{ }^{3}$ Surgical evacuation is the standard treatment in most symptomatic cases and most patients recover with a straightforward course, with clinical improvement occurring within the first 2 days. ${ }^{4,5}$ There is, however, a subset of the population that does not have resolution of preoperative symptoms or who develop new transient deficits postoperatively. These patients have a more protracted course and often require EEG monitor-

ABBREVIATIONS $\mathrm{CSDH}=$ chronic subdural hematoma; $D C=$ direct current; $E C O G=$ electrocorticography; $M G S=$ Markwalder Grading Scale; $S A H=$ subarachnoid hemorrhage; $\mathrm{SD}=$ spreading depolarization.

SUBMITTED October 27, 2019. ACCEPTED January 20, 2020.

INCLUDE WHEN CITING Published online March 27, 2020; DOI: 10.3171/2020.1.JNS192914. 
ing, additional imaging, or even re-evacuation of relatively minor residual hematoma. ${ }^{6-8}$ These deteriorations are often attributed to a variety of problems such as electrolyte abnormalities, infection, medications, focal seizure, or transient ischemic attack. ${ }^{9}$ However, these explanations seem unsatisfactory for a subset of patients. In the current study, we examine the hypothesis that a different pathophysiological mechanism not detectable by standard noninvasive monitoring (spreading depolarization [SD]) may contribute to neurological deficits in some cases.

SD is a well-described electrophysiological phenomenon in which massive waves of cellular depolarization cause transient neuronal dysfunction, resulting in transient depression of high-frequency electrocorticography (ECoG) activity (spreading depression). ${ }^{10,11}$ Electrical signaling is transiently silenced in SD due to loss of synaptic potentials. Recent clinical observations have definitively documented the presence of SD after many types of brain injury. ${ }^{12,13}$ It should be noted that in acute brain injury, patients are typically sedated during monitoring, precluding the observation of neurological SD correlates. SD is, however, believed to represent the physiological correlate of the scintillating scotoma in migraine auras as it crosses the calcarine cortex. ${ }^{14}$ Recurrent SD has also been closely linked to clinical neurological deterioration after aneurysmal subarachnoid hemorrhage (SAH). Dreier et al. demonstrated time-linked clinical deterioration in such patients with multiple SDs documented with ECoG.$^{12}$ In addition, SD has recently been documented to occur during elective neurosurgical procedures ${ }^{15}$ and as the likely causal event preceding some cases of clinical brain death. ${ }^{16,17}$

Based on these observations, we hypothesize that at least some instances of delayed neurological recovery after cSDH evacuation may be related to SD. Measurement of such events has historically been limited due to technical limitations that preclude SD detection from scalp recordings. The aim of this prospective study was to investigate the presence of SD in patients after cSDH evacuation, using invasive recording methods.

\section{Methods Study Design}

Patients requiring evacuation of cSDH were prospectively recruited for this observational study from June 2017 to August 2018. The experimental protocol and informed consent were approved by the local IRB.

\section{Participants}

Entry criteria for the study were diagnosis of $\mathrm{cSDH}$, and surgery recommended per the treating neurosurgeon. Informed consent for monitoring was obtained from all individuals or their legal power of attorney, if they were unable to consent due to their pathology.

\section{Interventions}

A small craniotomy or large burr hole was performed per our institution's standard practice. Following evacuation of a hematoma and hemostasis, a subdural drain and a subdural $1 \times 6$ strip electrode (Wyler, platinum, 4-mm diameter, 10-mm interval between electrode centers; In- tegra Epilepsy) were placed in the subdural space onto the frontal cortex and parallel to the subdural drain (Fig. 1 left). Following placement of the strip, the lead was externalized through the craniotomy and out the scalp. This technique has been previously described in brain injury monitoring protocols. ${ }^{10}$

The strip was then connected to a full-band direct current (DC) amplifier in a referential montage as described previously, and recorded using a component neuromonitoring system (Moberg Research, Inc.). ${ }^{18}$ The recordings were continued until the subdural drain was removed, based on clinical judgment, at which point the strip was also removed at the bedside with gentle traction. No intervention (pharmacological or otherwise) was performed based on the ECoG recordings, which were only reviewed after removal of the strip.

\section{Data Collection and Outcomes}

Data were exported to LabChart (AD Instruments) and scored using standard criteria of a slowly propagating DC shift accompanied by suppression of high-frequency (0.5$50 \mathrm{~Hz}$ ) signals (Fig. 1 right). ${ }^{10}$ Both referential and bipolar montages were used to aid in signal interpretation. Periods of excessive electrical noise were excluded from analysis. ECoG scoring was blinded to the clinical course.

Neurological deterioration was quantified with pre- and postoperative Markwalder Grading Scale (MGS) scores (Table 1). ${ }^{5,19,20}$ Any increase in score on the MGS was considered to be a deterioration. A patient's MGS score was recorded immediately postoperatively and on postoperative day 10, 1 month postoperatively, and 6 months postoperatively via phone interviews. The incidence of clinically required EEG recordings was also recorded as a further measure of neurological deterioration. A standard nursing protocol to examine four-extremity motor strength and verbal orientation every hour for the first 24-48 hours after surgery was also reviewed.

To assess for standard seizure and ECoG findings (nonSD), patients' recordings were reviewed in full by a boardcertified epileptologist (A.B.), who was provided de-identified patient files. A component neuromonitoring system reader (version Z.00.22, Moberg Research, Inc.) was used to import and review ECoG files. Patient files were characterized using the American Clinical Neurophysiology Society Standardized Critical Care EEG Terminology with a specific focus on presence of seizures. ${ }^{21}$

\section{Sample Size}

The incidence of SD in the postoperative period after cSDH evacuation in either normal or impaired patients was unknown; therefore, a power calculation could not be reliably estimated. Given the high prevalence of impaired recovery in the cSDH population and a historical rate of $50 \%-60 \%$ in the TBI population, we felt that a population of 40 patients would be sufficient in detecting a difference in occurrence of SD, if it were contributory. ${ }^{11}$ In order to address this issue of lack of power estimate, an interim analysis was performed after every 10 patients, both to review safety and to determine if SD frequency could be determined. 

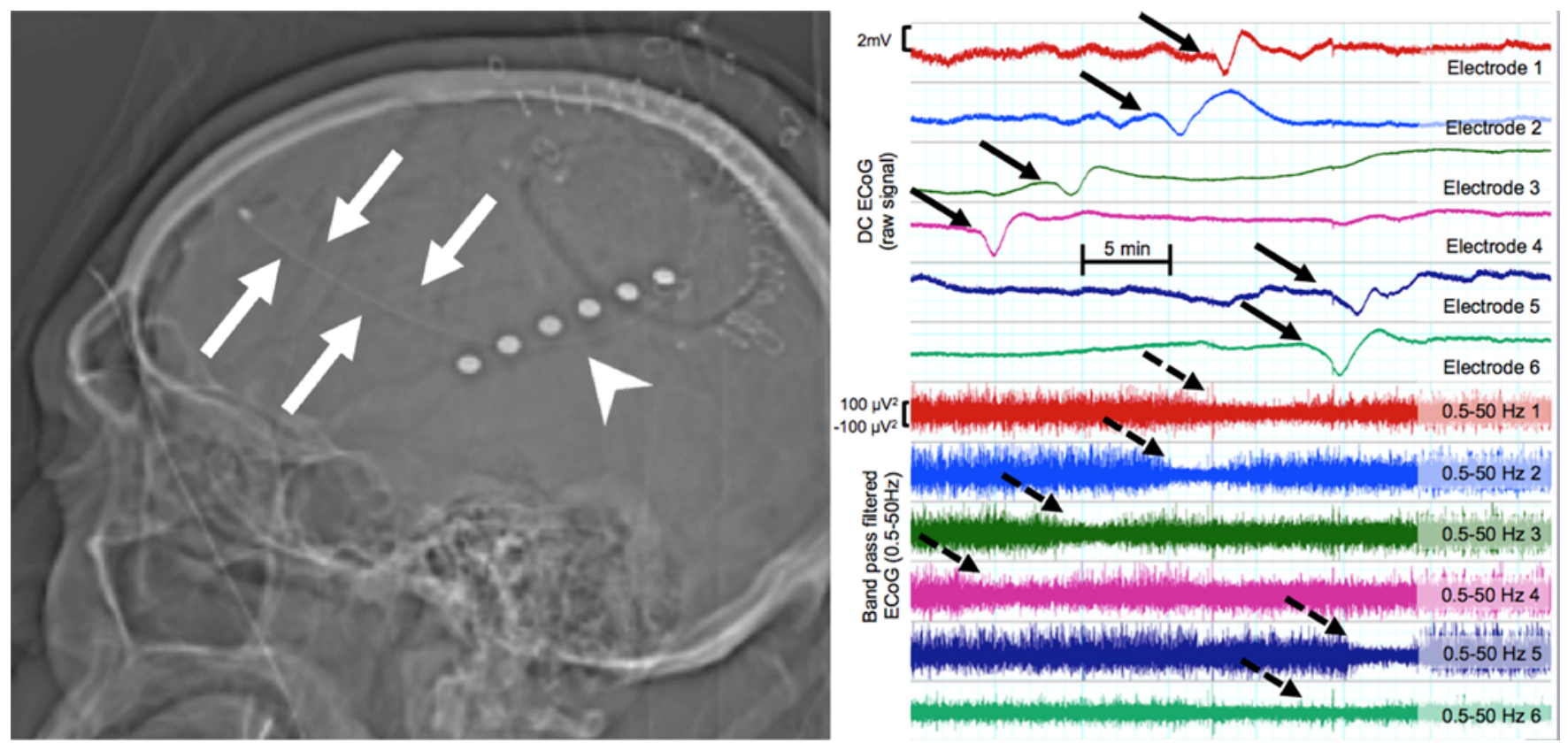

FIG. 1. Left: Cortical electrode placement. Subdural $1 \times 6$ platinum electrodes (arrowhead) were placed onto the cortex parallel to the subdural drain (arrows). Right: ECoG tracing of typical cortical SD. The upper 6 tracings show full-band ECoG to reveal the characteristic slow DC potential changes propagating between electrodes at approximately $2 \mathrm{~mm} / \mathrm{min}$ (arrows). The lower 6 tracings show data from the same electrodes but filtered at $0.5-50 \mathrm{~Hz}$, to emphasize the suppression of high-frequency activity during propagation of the same event (dashed arrows). The image is highly time-compressed, demonstrating a 35-minute interval, with one large box indicating 5 minutes. Figure is available in color online only.

\section{Blinding}

Clinicians reading the ECoG data were blinded to patient outcomes. Outcome assessors were blinded to the results of clinical recordings.

\section{Statistical Methods}

Descriptive statistics were used to characterize participants by demographics and other variables. Individuals for whom some measures were not applicable (such as side of electrode for unilateral cSDH) were excluded from that particular analysis. Patients who were lost to follow-up at postoperative MGS assessment $(\mathrm{n}=1$ at month 1 and $\mathrm{n}=13$ at month 6 ) were also omitted from those analyses. Potential confounding by age and sex was assessed in relationship to SD and other variables, but because those factors were not associated (Table 2), age and sex were not included in the analysis. The relationship between demographics, patient history, or subdural hematoma characteristics with SD, and the relationship of SD with MGS, EEG, or disposition were evaluated using Fisher's exact test due to cell sizes $\leq 5$. SD in relation to length of hospital stay (days) was evaluated using an independent 2-sample t-test. Those $p$ values $\leq 0.05$ were considered statistically significant. All analyses were conducted using SAS software (version 9.4).

\section{Results}

\section{Participants}

Data collection and ECoG analysis were completed on 40 patients (Table 2). The mean participant age was 72 years, and $30 \%$ were female. Three patients $(7.5 \%)$ had a history of a craniotomy and only 1 patient presented with seizure. All patients presented with an MGS score of either 1 or 2 . Most cSDHs (67.5\%) were unilateral, and in such cases the electrode was always placed on the side with the larger cSDH if surgery was bilateral. The mean volume of cSDH was $223.3 \mathrm{~cm}^{3}$ and midline shift was 5.54 $\mathrm{mm}$ (Table 3). Almost all patients had a small craniotomy ( $2-3 \mathrm{~cm}$ in diameter), with only 3 patients $(7.5 \%)$ having a simple burr hole.

\section{TABLE 1. Markwalder Grading Scale ${ }^{5}$}

\begin{tabular}{|c|c|c|}
\hline Grade & Neurological Findings & Mental Status \\
\hline 0 & $\begin{array}{l}\text { Normal (incidental/no symp- } \\
\text { toms) }\end{array}$ & Normal \\
\hline 1 & $\begin{array}{l}\text { None or mild } \\
\text { Reflex asymmetry: pronator drift; } \\
\quad \geq 4+\text { motor }\end{array}$ & $\begin{array}{l}\text { Fully alert and conscious; } \\
\text { may have mild symptoms } \\
\text { such as headache }\end{array}$ \\
\hline 2 & $\begin{array}{l}\text { Variable neurological deficits } \\
\text { such as hemiparesis }\end{array}$ & Drowsy or disoriented \\
\hline 3 & $\begin{array}{l}\text { Severe focal signs such as } \\
\text { hemiplegia }\end{array}$ & $\begin{array}{l}\text { Stuporous but responds } \\
\text { appropriately to noxious } \\
\text { stimuli }\end{array}$ \\
\hline 4 & $\begin{array}{l}\text { Decerebrate or decorticate } \\
\text { posturing }\end{array}$ & $\begin{array}{l}\text { Comatose, with absent mo- } \\
\text { tor responses to painful } \\
\text { stimuli }\end{array}$ \\
\hline
\end{tabular}


TABLE 2. Patient demographics

\begin{tabular}{lcccr}
\hline \multicolumn{1}{c}{ Characteristic } & $\begin{array}{c}\text { All Patients } \\
(\mathrm{n}=40)\end{array}$ & $\begin{array}{c}\text { No SD } \\
(\mathrm{n}=34)\end{array}$ & $\begin{array}{c}\text { SD } \\
(\mathrm{n}=6)\end{array}$ & $\begin{array}{c}\mathrm{p} \\
\text { Value* }\end{array}$ \\
\hline Female sex, \% & 30.0 & 66.6 & 33.3 & $>0.99$ \\
\hline Mean age, yrs & 71.8 & 71.5 & 73.3 & 0.85 \\
\hline Past medical history, \% & & & & \\
\hline$\quad$ Alcohol abuse history & 7.5 & 8.8 & 0.0 & $>0.99$ \\
\hline$\quad$ Seizure disorder & 5.0 & 2.9 & 16.7 & 0.28 \\
\hline Past surgical history, \% & & & & \\
\hline$\quad$ History of craniotomy & 7.5 & 5.9 & 16.7 & 0.39 \\
\hline Medications, \% & & & & \\
\hline$\quad$ Antiepileptic drug use & 5.0 & 2.9 & 16.7 & 0.28 \\
\hline$\quad$ Anticoagulation use & 30.0 & 26.5 & 50.0 & 0.34 \\
\hline Seizures on admission, \% & 2.5 & 2.9 & 0.0 & $>0.99$ \\
\hline
\end{tabular}

Demographic and clinical baseline characteristics of the population groups. * None of these variables significantly differed according to SD status when evaluated using the Fisher's exact test.

\section{ECoG Characteristics}

ECoG detection of SD in neurointensive care unit patients has become well established in recent years, but this was the first application to cSDH surgical patients, and theoretical technical challenges (such as patient movement, relatively short recording window, potential lack of direct electrode strip contact due to atrophy) were unknown..$^{10}$ All 40 patients had adequate quality ECoG recordings appropriate for SD detection. The mean duration of readable ECoG recordings was 39 hours and 46 minutes \pm 15 hours and 45 minutes, for a total recording duration of 1591 hours and 5 minutes in these 40 patients (Table 4). SD was definitively detected in 6 (15\%, range 2-40 SDs) of the 40 patients (Fig. 2). The recording duration of valid data in these patients was slightly longer (mean 48 hours and 19 minutes vs 38 hours and 16 minutes), but this difference was not significant $(\mathrm{p}=0.15)$. The rate was $0.25 \mathrm{SD}$ events per hour of recording, which is similar to rates reported in patients with acute neurological injuries. ${ }^{22}$ ECoG depression duration, believed to represent a measure of the health of tissue and ability to respond to the metabolic load of
$\mathrm{SD},{ }^{10}$ was expectedly shorter (mean 5 minutes and $3 \mathrm{sec}$ onds per SD) than typically seen after acute neurological injury, in which depression duration in the context of ischemic or traumatic injury can be more than 20 minutes or even permanent. ${ }^{23}$

\section{Outcomes}

Overall length of stay averaged 7.5 days and most patients $(60 \%)$ were discharged to a rehabilitation facility (Table 5). Two patients (5\%) had early clinical deterioration, as assessed by the MGS during the hospitalization, both of whom also had definitive SD recorded (Table 6). An EEG was ordered in 6 patients (15\%): 3 for decline in neurological examination, 2 for altered mental status, and 1 for persistent neurological deficit, although no seizure or epileptiform activity was observed in any patient on clinical EEG. Seizure prophylaxis was decided by the treating physician per standard of care, with 36 (90\%) receiving some dose of levetiracetam $(56.4 \%$ received 1000 mg orally twice a day). Three patients (7.5\%) had delayed recurrence of the cSDH, requiring re-evacuation. Four additional patients required reoperation: 2 for infection, 1 for acute recurrence after a fall, and 1 for a small postoperative acute subdural hematoma evacuated due to persistent deficit. There was one monitoring complication, which was the finding of a retained fragment of the electrode strip. This patient was 1 of the 3 patients with recurrent $\mathrm{cSDH}$, and the fragment was removed from the subgaleal space at the second procedure. This did not result in any harmful consequence to the patient and was not thought to be related to the recurrence due to it being located in the subgaleal space. A placement and removal protocol change was initiated after this event, which had not been observed in our previous ECoG studies or since. ${ }^{18,24,25}$ Two patients died prior to their 1-month postoperative followup, 1 with SD and 1 without. We did not record the cause of death in this pilot study.

\section{Comparison of Patients With and Without SD}

Overall, baseline characteristics were similar between patients with and without SD (Tables 2, 3, and 6). The volume of cSDH was similar, and preoperative neurological examinations were essentially identical, with approxi-

TABLE 3. Chronic subdural hematoma characteristics

\begin{tabular}{lcccc}
\hline \multicolumn{1}{c}{ Characteristic } & $\begin{array}{c}\text { All Patients } \\
(\mathrm{n}=40)\end{array}$ & $\begin{array}{c}\text { No SD } \\
(\mathrm{n}=34)\end{array}$ & $\begin{array}{c}\text { SD } \\
(\mathrm{n}=6)\end{array}$ & p Value \\
\hline Unilateral subdural hematoma & $67.5 \%$ & $67.6 \%$ & $66.7 \%$ & 1.0 \\
\hline Mean preop CT density, $\mathrm{HU}$ & 38.6 & 38.5 & 39.1 & 0.73 \\
\hline Mean volume of SDH, $\mathrm{cm}^{3}$ & 223.3 & 229.2 & 190.8 & 1.0 \\
\hline Mean midline shift, $\mathrm{mm}$ & 5.54 & 5.75 & 4.42 & 0.83 \\
\hline Burr hole surgery (mutually exclusive w/ craniotomy) & $7.5 \%$ & $8.8 \%$ & $0.0 \%$ & 1.0 \\
\hline Side of electrode (rt) & $50.0 \%$ & $62.5 \%$ & $0.0 \%$ & 0.44 \\
\hline Mean postop pneumocephalus, $\mathrm{cm}^{3}$ & 31.7 & 31.9 & 30.3 & 0.91 \\
\hline
\end{tabular}

$\mathrm{HU}=$ Hounsfield units.

None of these variables significantly differed according to SD status when evaluated using Fisher's exact test for cell sizes $\leq 5$. 
TABLE 4. Summary of SD data

\begin{tabular}{ll}
\hline \multicolumn{1}{c}{ Variable } & \multicolumn{1}{c}{ Value } \\
\hline Total valid recorded time & $1591 \mathrm{hrs}, 5 \mathrm{~min}$ (93\% of total time w/ electrode) \\
\hline Mean recording time per patient & $39 \mathrm{hrs}, 46 \mathrm{~min} \pm 15 \mathrm{hrs}, 45 \mathrm{~min}$ \\
\hline Overall occurrence of any SD & $6 / 40(15 \%)$ \\
\hline In 6 patients w/ SD & 0.25 (range 0.02-0.62) \\
\hline Mean rate of SD per hr & 11 (range 2-40) \\
\hline Mean no. of SDs & $43 \mathrm{~min}, 44 \mathrm{sec}$ (range 3 min to 3 hrs, $1 \mathrm{~min})$ \\
\hline Mean total depression duration & $1 \mathrm{~min}, 5 \mathrm{sec}$ (range $5 \mathrm{sec}$ to 2 min, 53 sec) \\
\hline Mean hourly depression duration per patient & $5 \mathrm{~min}, 3 \mathrm{sec}$ (range 2-28 min) \\
\hline Mean depression duration per SD & $28 \mathrm{hrs}$ (range 4-78 hrs) \\
\hline Mean time to first SD from surgery &
\end{tabular}

mately $33 \%$ with MGS scores of 2 and approximately $66 \%$ with MGS scores of 3 in both groups. Despite this baseline similarity, we found a significantly higher rate of neurological deterioration in patients who had SD (Fig. 3). Fifty percent of patients with SD experienced deterioration by at least 1 point on the MGS at any follow-up interval compared to only $8.8 \%$ of those without SD $(p=0.03)$. There was a trend toward more early deterioration in patients with SD as well, although not significant. Similarly, there was a trend toward worse discharge disposition in patients with SD (none were discharged home, while $27 \%$ of those without SD were discharged home), although this was not significant (Table 5).

\section{Time Course of Neurological Deficits and SD}

Only 2 patients experienced deterioration by at least one grade on the MGS during hospitalization, and both of the patients were found to have SD (Fig. 2). One patient's new motor deficit with increased somnolence was observed 40 hours following the appearance of a cluster of 6 SDs, after the electrode strip was removed. It is unknown if the deficit was related to SD; however, the patient had an EEG performed, which did not demonstrate seizures and also showed no new infarct on imaging, so the cause of the deficit was otherwise unexplained. The other patient, however, demonstrated a time-locked (i.e., the deficit started at exactly the same time as an SD was

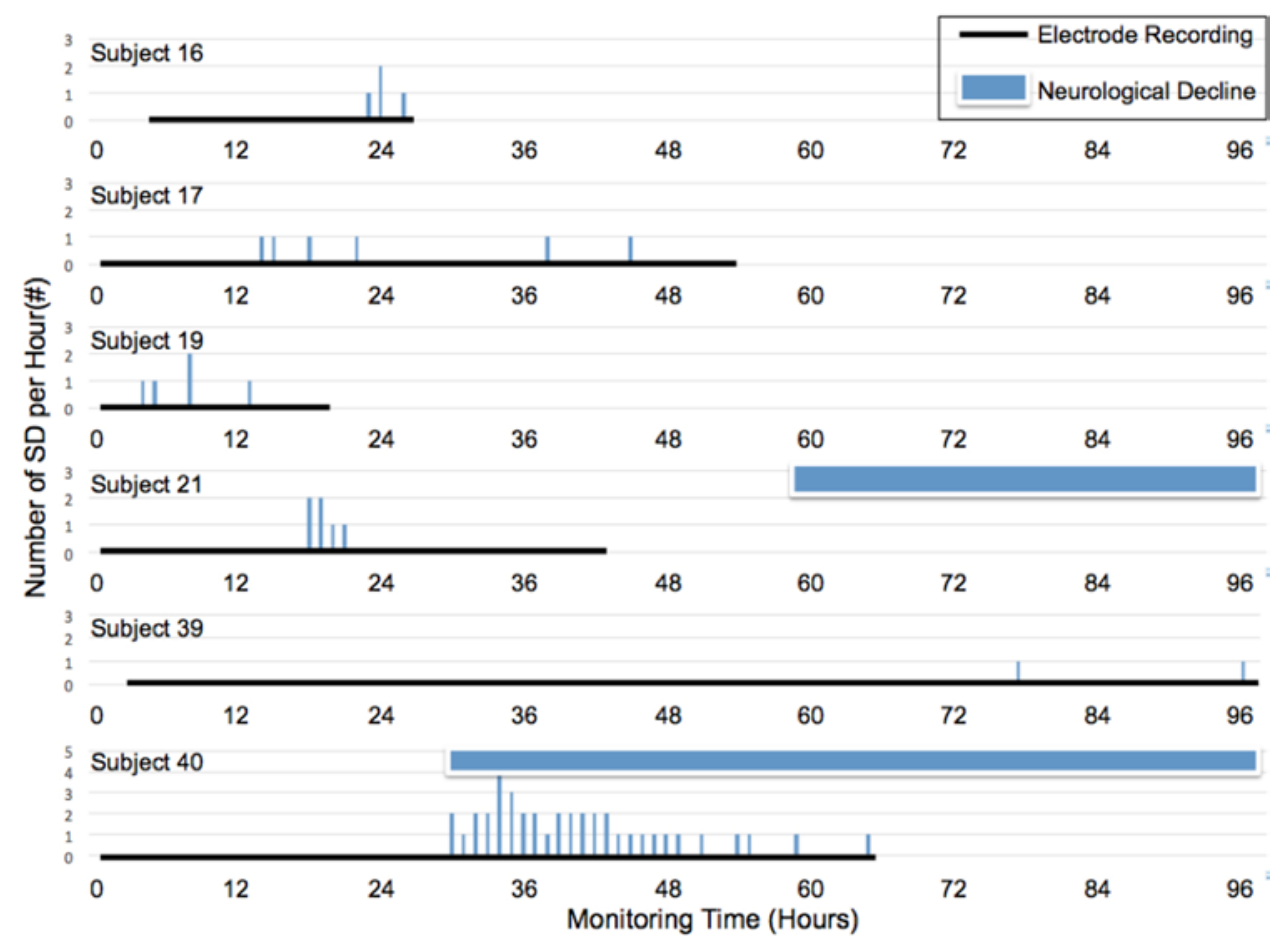

FIG. 2. Plot of frequency of SD since the time of surgery completion (represented by 0 on the $x$-axis). A rectangular block (in blue) represents neurological decline of the patient. The solid black line superimposed over the $x$-axis demonstrates the duration of electrode recording. Figure is available in color online only. 
TABLE 5. Early outcomes

\begin{tabular}{lcccc}
\hline \multicolumn{1}{c}{ Characteristic } & $\begin{array}{c}\text { All Patients } \\
(\mathrm{n}=40)\end{array}$ & $\begin{array}{c}\text { No SD } \\
(\mathrm{n}=34)\end{array}$ & $\begin{array}{c}\text { SD } \\
(\mathrm{n}=6)\end{array}$ & $\mathrm{p}$ Value \\
\hline Mean hospital LOS, days & 7.5 & 6.9 & 7.6 & 0.06 \\
\hline Disposition, \% & & & & 0.35 \\
\hline Home & 27.5 & 32.3 & 0.0 & \\
\hline Rehabilitation & 60.0 & 55.9 & 83.3 & \\
\hline Skilled nursing facility & 10.0 & 8.8 & 16.7 & \\
\hline Hospice & 2.5 & 2.9 & 0.0 & \\
\hline
\end{tabular}

LOS = length of stay.

$\mathrm{SD}$ is the exposure and postoperative course variables are the outcomes. Disposition did not significantly differ by SD status, as evaluated using Fisher's exact test. Length of hospital stay in days also did not significantly differ, using an independent 2-sample t-test.

seen on ECoG recording) new focal neurological deficit (aphasia and facial droop) at the start of a series of multiple clusters of SD (Fig. 4). Again, EEG and MRI findings did not explain the deficit. There was a very small area of infarct noted (Fig. 4, dashed circle), thought to be related to a small cortical vessel that was coagulated at the time of surgery. The patient underwent re-evacuation of a small postoperative acute SDH after 3 days due to this new deficit without other explanation, but the deficit did not improve. The electrode strip was removed at the time of surgery, so the further time course of SD is unknown. The deficit gradually improved and the patient recovered completely by day 11 .

\section{Analysis of Seizures on ECoG and EEG}

Thirty-eight of the 40 patients' files could be reviewed and characterized by the epileptologist. Two (5.3\%) of the
38 patients had seizures appreciated on the ECoG strip. Neither of these patients had a clinical EEG ordered during their admission. Both had MGS scores of 1 preoperatively that remained 1 on postoperative days 1 and 10 . No other seizures were noted in any patient during the monitoring duration. Of the 5 patients (12.5\%) who had standard EEG ordered for clinical reasons during the hospitalization, 2 were also found to have SD.

\section{Discussion}

These data represent the first observation of SD occurring after $\mathrm{cSDH}$ evacuation. SD has been recognized to cause transient neurological deficits and is therefore an area of interest in patients with cSDH who may have a protracted recovery following subdural evacuation, despite adequate drainage. ${ }^{6,726} \mathrm{SD}$ has been identified as being present across a spectrum of neurological diseases, from the benign visual auras of migraines, to stroke and worsening ischemia in patients with $\mathrm{SAH} \cdot{ }^{13,14,23}$ In addition, SD has recently been documented to occur after elective neurosurgical procedures. ${ }^{15}$ This is relevant because although SD in cSDH may not result in long-term harm (similar to migraines), resultant transient deficits can delay recovery and potentially result in unnecessary workup or even reoperation. In other words, SD after cSDH may not be directly harmful to brain tissue compared to cases of ischemia, where mounting evidence supports a deleterious role in infarct development. ${ }^{13,16}$ Conversely, if SD is related to transient fluctuating neurological deficits, there may be a secondary relationship with poor outcomes, although our current preliminary data are underpowered to detect any such difference.

Our first observation was that we were able to demonstrate the feasibility of recording reliable ECoG in this patient population, despite widened subdural spaces and

TABLE 6. Neurological outcomes during follow-up

\begin{tabular}{|c|c|c|c|c|c|c|c|}
\hline \multirow[b]{2}{*}{ Interval } & \multicolumn{5}{|c|}{ MGS Score (\%) } & \multirow[b]{2}{*}{ Death (\%) } & \multirow[b]{2}{*}{$p$ Value } \\
\hline & 0 & 1 & 2 & 3 & 4 & & \\
\hline \multicolumn{8}{|l|}{ Preop } \\
\hline No SD, $n=34$ & 0.0 & 32.3 & 67.7 & 0.0 & 0.0 & 0.0 & \\
\hline $\mathrm{SD}, \mathrm{n}=6$ & 0.0 & 33.3 & 66.7 & 0.0 & 0.0 & 0.0 & 1.0 \\
\hline \multicolumn{8}{|l|}{ Postop day 1} \\
\hline No SD, $n=34$ & 47.1 & 29.4 & 23.5 & 0.0 & 0.0 & 0.0 & \\
\hline$S D, n=6$ & 66.7 & 0.0 & 33.3 & 0.0 & 0.0 & 0.0 & 0.39 \\
\hline \multicolumn{8}{|l|}{ Postop day 10} \\
\hline No SD, $n=34$ & 50.0 & 38.2 & 11.8 & 0.0 & 0.0 & 0.0 & \\
\hline$S D, n=6$ & 66.7 & 0.0 & 16.7 & 16.7 & 0.0 & 0.0 & 0.06 \\
\hline \multicolumn{8}{|l|}{ Postop 1 mo } \\
\hline No SD, $n=33$ & 51.5 & 42.4 & 3.0 & 0.0 & 0.0 & 3.0 & \\
\hline$S D, n=6$ & 50.0 & 33.3 & 0.0 & 0.0 & 0.0 & 16.7 & 0.52 \\
\hline \multicolumn{8}{|l|}{ Postop 6 mos } \\
\hline No SD, $n=21$ & 57.1 & 38.1 & 4.8 & 0.0 & 0.0 & 0.0 & \\
\hline $\mathrm{SD}, \mathrm{n}=5$ & 80.0 & 0.0 & 0.0 & 0.0 & 0.0 & 20.0 & 0.13 \\
\hline
\end{tabular}




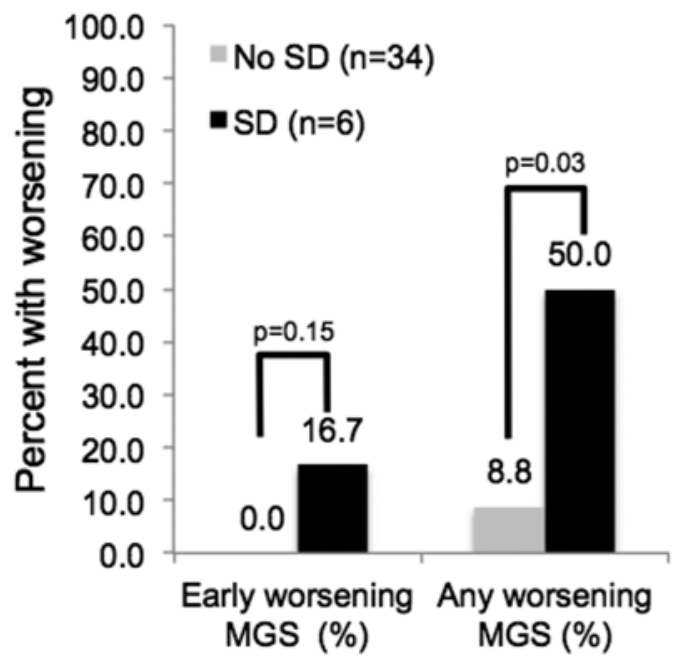

FIG. 3. Comparison between patients without and with SD regarding whether examination by the MGS worsened early (preoperative vs postoperative day 1) or late (preoperative vs worsening at any delayed time point up to 6 months). Both categories demonstrated a trend toward more worsening in the SD group, and late worsening was significantly more likely in patients with SD, according to Fisher's exact test.

the presence of postoperative pneumocephalus. Second, SD was definitively detected in $6(15 \%)$ of the 40 patients, ranging from 2 to 40 SDs during the relatively short recording period. This number may be underreported due to the limited detection area of the strip electrode. Our data support the hypothesis that SD may be a newly defined mechanism for delayed neurological deficits after cSDH evacuation, because there was a significantly higher rate of delayed deterioration in patients with SD compared to those without. In 1 case, there was a time-locked focal neurological deficit with onset of multiple SDs (Fig. 4).

The clinical problem of cSDH is enormous and growing with an aging population. ${ }^{3}$ The problem of fluctuating deficits after surgical evacuation has been well characterized $^{6,7}$ and represents a clinically frustrating problem. The underlying pathogenesis of these events has previously been hypothesized to be related to $\mathrm{SD}^{7}$, but due to technical limitations, it has been impossible to definitively record SD until recently. ${ }^{10}$ Using techniques initially studied in acute neurological injuries, ${ }^{18}$ we were able to support this proposed mechanism in this study.

Given the relevance of SD in cases of severe acute injuries in lesion progression, recent intense interest in targeted therapies for SD has developed. ${ }^{22,24,27} \mathrm{~N}$-methyl-D-aspartate-receptor antagonism with ketamine has been the most promising candidate; a recent pilot trial we conducted demonstrated a significant reduction in SD in patients receiving ketamine at a dose $>1.15 \mathrm{mg} / \mathrm{kg} / \mathrm{hr} .^{24}$ Although ketamine may not be the optimal target in this relatively elderly population due to its sedating effects, other agents with similar effects are under investigation. This could potentially have a significant impact if delayed recovery after cSDH could be reduced. In addition to the economic burden of negative workups (with MRI and EEG testing), there are likely some cases of repeat surgery for relatively minor residual or recurrent cSDH that are performed due to such deficits. The case of time-locked deterioration in this series demonstrates this point, in which the patient was taken back to the operating room for a relatively minor residual hematoma without recovery. This suggests that deterioration was not, in fact, due to the mass effect from the hematoma, but rather was due to the SD. We did not review the ECoG data in real time so that the findings

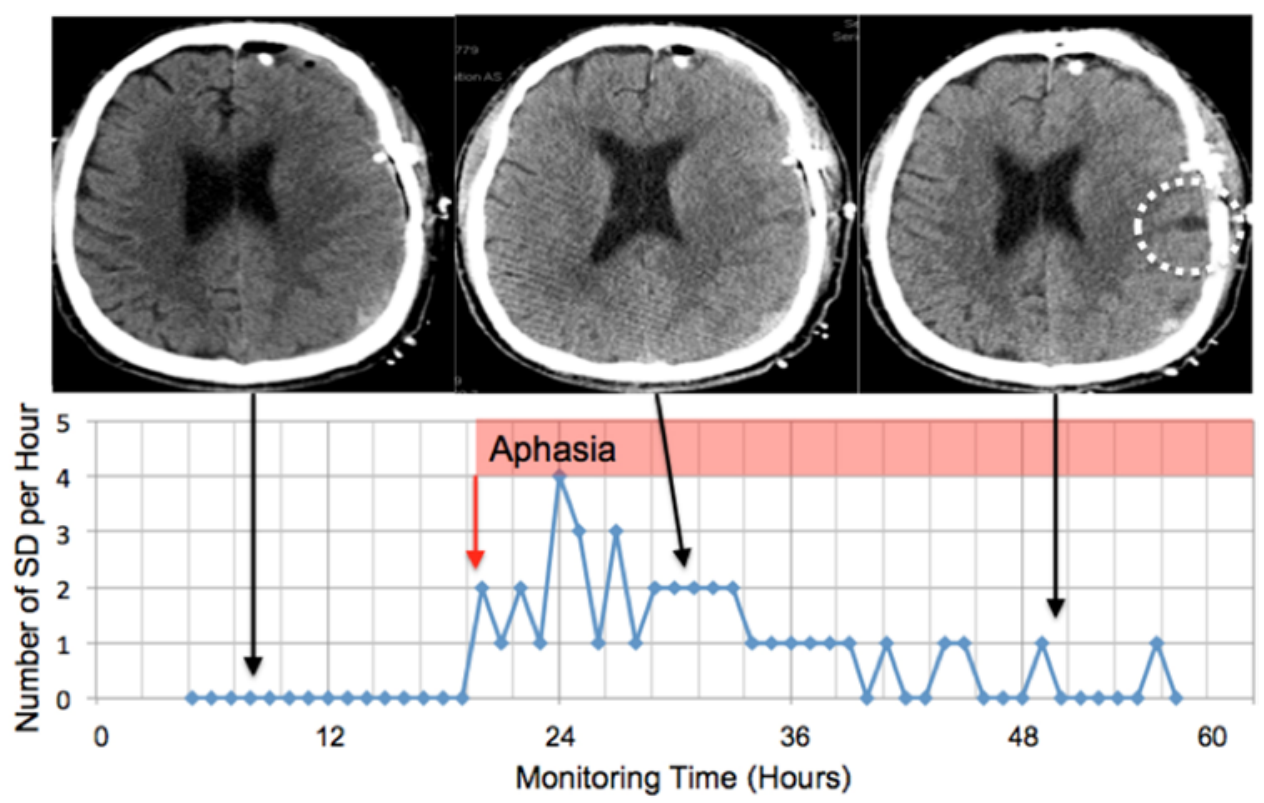

FIG. 4. Time course of patient with clinical deterioration time-locked with SD. The upper 3 images represent CT scans performed at various intervals. Note the lack of significant residual cSDH on the left. A small area of ischemia (dashed circle) is noted, but was not believed to explain the patient's deficit. The graph shows the number of SDs per hour during the recording time. There was time-locked onset of aphasia with the first SD event. Figure is available in color online only. 
would not influence management; therefore, the presence of SD was unknown in this case until after surgery.

Although 3 patients had "asymptomatic" SD, we hypothesize that this could be related to the nature of the clinical assessments made. Because these strips were typically placed over the frontal lobe for practical reasons, deficits associated with transient synaptic suppression in the frontal lobe could be missed by a gross assessment tool such as the MGS. Localized SD in 1 frontal lobe would not be expected to lead to a measurable deficit unless motor or language regions were involved, but could potentially have relevance in other cognitive recovery after cSDH evacuation.

It should also be noted that no patients with SD were discharged home, which demonstrated a provocative trend, even if it did not reach statistical significance. In addition, we noted more deterioration at any time point in the patients with SD compared to those without. These results are, however, primarily exploratory and will require a larger study focused on outcome to confirm. It is also interesting to note that only 2 patients demonstrated seizures on ECoG, neither of whom had any change in neurological status. Given that seizure treatment was performed per standard of care, it is certainly possible that more patients would have had seizures without treatment, but the lack of clinical correlates in these 2 cases suggests that compared to SD, seizure may be less relevant as a cause of fluctuating deficits.

\section{Limitations}

There are some limitations to the current study. It is unknown if the cSDH itself or the surgical procedure is a larger risk for occurrence of SD. Because there are no validated noninvasive methods to detect SD, all patients required surgical procedures. It is possible that the enrollment in the prospective study affected other variables, such as increased attention during the surgical procedure and more attentive postoperative care, which could also have potentially affected SD occurrence or other factors leading to deterioration. It is also possible that some of the patients with persistent deficits or late EEG requirements had SDs that were unable to be detected because of strip positioning or the relatively short monitoring window. These data are preliminary and certainly no directly causal relationship is established, but this is the first observation that SD can occur after cSDH evacuation and seems to be associated with delayed neurological deterioration.

\section{Future Studies}

Future studies are needed to test the hypothesis that targeted treatment of SD could improve recovery after SDH evacuation and would have an important potential to improve outcomes in this elderly and growing population. While ketamine has been shown in brain injury to effectively suppress SD, the potential for sedation does not make this drug an ideal candidate for first-line treatment in this population. ${ }^{24}$ Multiple migraine prophylactic medications, such as topiramate and gabapentin, have been suggested to act via an anti-SD mechanism in mouse models. ${ }^{28,29}$ Pretreatment with these medications may therefore be feasible in cases of cSDH, where there is typically less urgency for immediate action, compared to acute brain injury.

\section{Conclusions}

This is the first observation of SD occurring after cSDH evacuation, at a rate of $15 \%$. Furthermore, SD was associated with neurological deterioration. These findings support the hypothesis that SD may be a physiological underpinning of otherwise unexplained fluctuating neurological deficit after cSDH evacuation. This may represent a new therapeutic target in these patients, and SD-targeted therapies could be tested in prospective clinical trials.

\section{Acknowledgments}

Financial support for this study was provided by the University of New Mexico Department of Neurosurgery.

\section{References}

1. Almenawer SA, Farrokhyar F, Hong C, et al. Chronic subdural hematoma management: a systematic review and metaanalysis of 34,829 patients. Ann Surg. 2014;259(3):449-457.

2. Frontera JA, Egorova N, Moskowitz AJ. National trend in prevalence, cost, and discharge disposition after subdural hematoma from 1998-2007. Crit Care Med. 2011;39(7):16191625.

3. Balser D, Farooq S, Mehmood T, et al. Actual and projected incidence rates for chronic subdural hematomas in United States Veterans Administration and civilian populations. $J$ Neurosurg. 2015;123(5):1209-1215.

4. Iliescu IA. Current diagnosis and treatment of chronic subdural haematomas. J Med Life. 2015;8(3):278-284.

5. Markwalder TM, Steinsiepe KF, Rohner M, et al. The course of chronic subdural hematomas after burr-hole craniostomy and closed-system drainage. J Neurosurg. 1981;55(3):390396.

6. Kaminski HJ, Hlavin ML, Likavec MJ, Schmidley JW. Transient neurologic deficit caused by chronic subdural hematoma. Am J Med. 1992;92(6):698-700.

7. Moster ML, Johnston DE, Reinmuth OM. Chronic subdural hematoma with transient neurological deficits: a review of 15 cases. Ann Neurol. 1983;14(5):539-542.

8. Rabinstein AA, Chung SY, Rudzinski LA, Lanzino G. Seizures after evacuation of subdural hematomas: incidence, risk factors, and functional impact. J Neurosurg. 2010;112(2):455460.

9. Rovlias A, Theodoropoulos S, Papoutsakis D. Chronic subdural hematoma: surgical management and outcome in 986 cases: A classification and regression tree approach. Surg Neurol Int. 2015;6:127.

10. Dreier JP, Fabricius M, Ayata C, et al. Recording, analysis, and interpretation of spreading depolarizations in neurointensive care: review and recommendations of the COSBID research group. J Cereb Blood Flow Metab. 2017;37(5):15951625.

11. Lauritzen M, Dreier JP, Fabricius M, et al. Clinical relevance of cortical spreading depression in neurological disorders: migraine, malignant stroke, subarachnoid and intracranial hemorrhage, and traumatic brain injury. J Cereb Blood Flow Metab. 2011;31(1):17-35.

12. Dreier JP, Woitzik J, Fabricius M, et al. Delayed ischaemic neurological deficits after subarachnoid haemorrhage are associated with clusters of spreading depolarizations. Brain. 2006;129(Pt 12):3224-3237.

13. Hartings JA, Shuttleworth CW, Kirov SA, et al. The continuum of spreading depolarizations in acute cortical lesion development: examining Leão's legacy. J Cereb Blood Flow Metab. 2017;37(5):1571-1594.

14. Hadjikhani N, Sanchez Del Rio M, Wu O, et al. Mechanisms 
of migraine aura revealed by functional MRI in human visual cortex. Proc Natl Acad Sci U S A. 2001;98(8):4687-4692.

15. Carlson AP, Shuttleworth CW, Mead B, et al. Cortical spreading depression occurs during elective neurosurgical procedures. J Neurosurg. 2017;126(1):266-273.

16. Carlson AP, Shuttleworth CW, Major S, et al. Terminal spreading depolarizations causing electrocortical silencing prior to clinical brain death: case report. J Neurosurg. 2018;131(6):1773-1779.

17. Dreier JP, Major S, Foreman B, et al. Terminal spreading depolarization and electrical silence in death of human cerebral cortex. Ann Neurol. 2018;83(2):295-310.

18. Hartings JA, Li C, Hinzman JM, et al. Direct current electrocorticography for clinical neuromonitoring of spreading depolarizations. J Cereb Blood Flow Metab. 2017;37(5):18571870.

19. El-Kadi H, Miele VJ, Kaufman HH. Prognosis of chronic subdural hematomas. Neurosurg Clin N Am. 2000;11(3):553567.

20. Thomas PAW, Mitchell PS, Marshman LAG. Early postoperative morbidity after chronic subdural hematoma: predictive usefulness of the physiological and operative severity score for enumeration of mortality and morbidity, American College of Surgeons National Surgical Quality Improvement Program, and American Society of Anesthesiologists grade in a prospective cohort. World Neurosurg. 2019;124:e489e497.

21. Hirsch LJ, LaRoche SM, Gaspard N, et al. American Clinical Neurophysiology Society's Standardized Critical Care EEG Terminology: 2012 version. J Clin Neurophysiol. 2013;30(1):1-27.

22. Hertle DN, Dreier JP, Woitzik J, et al. Effect of analgesics and sedatives on the occurrence of spreading depolarizations accompanying acute brain injury. Brain. 2012;135(Pt 8):2390-2398

23. Nakamura H, Strong AJ, Dohmen C, et al. Spreading depolarizations cycle around and enlarge focal ischaemic brain lesions. Brain. 2010;133(Pt 7):1994-2006.

24. Carlson AP, Abbas M, Alunday RL, et al. Spreading depolarization in acute brain injury inhibited by ketamine: a prospective, randomized, multiple crossover trial. J Neurosurg. 2019;130(5):1513-1519.

25. Drenckhahn C, Windler C, Major S, et al. Complications in aneurysmal subarachnoid hemorrhage patients with and without subdural electrode strip for electrocorticography. J Clin Neurophysiol. 2016;33(3):250-259.
26. Levesque M, Iorio-Morin C, Bocti C, et al. Nonepileptic, stereotypical, and intermittent symptoms (NESIS) in patients with subdural hematoma: proposal for a new clinical entity with therapeutic and prognostic implications [published online September 25, 2019]. Neurosurgery. doi:10.1093/neuros/ nyz355

27. Helbok R, Hartings JA, Schiefecker A, et al. What should a clinician do when spreading depolarizations are observed in a patient? Neurocrit Care. 2020;32(1):306-310.

28. Ayata C, Jin H, Kudo C, et al. Suppression of cortical spreading depression in migraine prophylaxis. Ann Neurol. 2006;59(4):652-661.

29. Hoffmann U, Dileköz E, Kudo C, Ayata C. Gabapentin suppresses cortical spreading depression susceptibility. J Cereb Blood Flow Metab. 2010;30(9):1588-1592.

\section{Disclosures}

Dr. Carlson reports direct stock ownership in Cerebroscope.

\section{Author Contributions}

Conception and design: Carlson, Mohammad. Acquisition of data: Carlson, Mohammad, Abbas, Ahmadian. Analysis and interpretation of data: Carlson, Mohammad, Ahmadian, Bhat. Drafting the article: Carlson, Mohammad, Bhat. Critically revising the article: Carlson, Shuttleworth. Reviewed submitted version of manuscript: all authors. Approved the final version of the manuscript on behalf of all authors: Carlson. Statistical analysis: Hill. Study supervision: Carlson.

\section{Supplemental Information}

\section{Previous Presentations}

Portions of this paper were previously presented as an oral presentation at the AANS Annual Meeting, April 17, 2019, in San Diego, California; as an oral presentation at the International Conference on Spreading Depolarizations, September 22, 2018, in Boca Raton, Florida; and as a plenary session at the AANS Annual Meeting, April 28, 2018, in New Orleans, Louisiana.

\section{Correspondence}

Andrew P. Carlson: University of New Mexico School of Medicine, Albuquerque, NM. andrewcarlson@salud.unm.edu. 